\title{
Síntese e caracterização de fosfatos de cálcio a partir da casca de ovo de galinha
}

\author{
(Synthesis and characterization of calcium \\ phosphates produced from chicken eggshell)
}

\author{
L. C. Gomes ${ }^{1}$, B. C. Di Lello', J. B. Campos ${ }^{2,3}$, M. Sampaio ${ }^{2}$ \\ ${ }^{1}$ Curso de Farmácia, ${ }^{2}$ Departamento de Engenharia Elétrica, Universidade Estácio de Sá, Rio de Janeiro, RJ \\ ${ }^{3}$ Departamento de Engenharia Mecânica, UERJ, Rio de Janeiro, $R J$ \\ lcgomes_2@yahoo.com.br,bruno.di.lello@live.estacio.br,brant@uerj.br, \\ marilzasa@oi.com.br
}

\begin{abstract}
Resumo
Fosfatos de cálcio são compostos cerâmicos atualmente utilizados para o desenvolvimento de biomaterais que atuam como substitutos ósseos nas áreas da ortopedia e odontologia clínica. Este trabalho teve como objetivo explorar rotas químicas para a conversão de casca de ovo de galinha, uma fonte rica em $\mathrm{CaCO}_{3}$, em fosfatos de cálcio inorgânicos, como o beta tricálcio fosfato $\left(\beta-\mathrm{Ca}_{3}\left(\mathrm{PO}_{4}\right)_{2}\right)$ e hidroxiapatita $\left(\mathrm{Ca}_{10}\left(\mathrm{PO}_{4}\right)_{6}(\mathrm{OH})_{2}\right)$ para a utilização como substitutos ósseos. Uma análise termodinâmica preliminar permitiu estabelecer quais os ácidos apresentam viabilidade reacional para o ataque químico à casca do ovo. Os sais inorgânicos produzidos, a partir do ataque ácido, utilizando

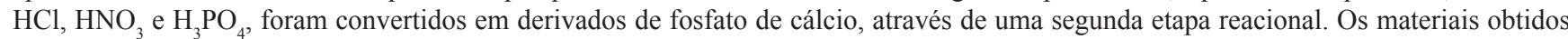
foram aquecidos em forno elétrico por $2 \mathrm{~h}$ a $900{ }^{\circ} \mathrm{C}$. A análise por difração de raios X mostrou misturas de diferentes fases de fosfatos de cálcio e também a obtenção de $\beta-\mathrm{Ca}_{3}\left(\mathrm{PO}_{4}\right)_{2}$ como fase única, de acordo com a rota e procedimentos de síntese adotados.
\end{abstract}

Palavras-chave: biomateriais, fosfatos de cálcio, beta tricálcio fosfato, substitutos ósseos.

\begin{abstract}
Calcium phosphates are ceramic compounds employed nowadays in development of biomaterials used as bone substitutes in clinical orthopedic and odontologic areas. The aim of this work was explore chemical routes able to convert chicken eggshell, a rich material in $\mathrm{CaCO}_{3}$, in inorganic calcium phosphates, as beta tricalcium phosphate $\left(\beta-\mathrm{Ca}_{3}\left(\mathrm{PO}_{4}\right)_{2}\right)$ and hydroxyapatite $\left(\mathrm{Ca}_{10}(\mathrm{PO})_{6}(\mathrm{OH})_{2}\right)$, materials $_{2}$ used as bone substitutes. A preliminary thermodynamic analysis has shown which acids were viable to chemical attack in chicken eggshell. Inorganic salts produced from chemical acid attack, using $\mathrm{HCl}, \mathrm{HNO}_{3}$ and $\mathrm{H}_{3} \mathrm{PO}_{4}$, were converted in calcium phosphates in a second reaction step. Materials produced were heated in electrical furnace over $2 \mathrm{~h}$ at $900{ }^{\circ} \mathrm{C}$. X-ray diffraction analyses have shown the presence of a mixture of different calcium phosphates phases and the formation of $\beta$-Ca $a_{3}\left(\mathrm{PO}_{4}\right)_{2}$ single phase from different chemical routes and synthesis procedures that have been adopted.
\end{abstract}

Keywords: biomaterials, calcium phosphates, beta tricalcium phosphate, bone substitutes.

\section{INTRODUÇÃO}

Os ossos e dentes de todos os vertebrados são compósitos naturais formados por moléculas de colágeno ligadas em cadeias lineares arranjadas em fibras. Entre estas moléculas há pequenos compartimentos intersticiais regularmente espaçados, onde estão presentes nanocristais de um sólido inorgânico, que representa $65 \%$ da massa total do osso [1].

Os fosfatos de cálcio apresentam-se hoje como os principais compostos estudados e empregados como biomateriais para a reposição e regeneração do tecido ósseo, pois apresentam como principais características: semelhança com a fase mineral de ossos; dentes e tecidos calcificados; excelente biocompatibilidade; bioatividade; ausência de toxicidade local ou sistêmica; ausência de resposta a corpo estranho ou inflamações; aparente habilidade em se ligar ao tecido hospedeiro; taxas de degradação variáveis; osteocondutividade (indicam o caminho para o crescimento ósseo, fazendo que ocorra sobre a superfície ou através dos poros). Dentre as cerâmicas de fosfato de cálcio, a hidroxiapatita (HA) se apresenta como material de referência na área de biomateriais [1-7]. Durante décadas, a HA foi a única biocerâmica do sistema dos fosfatos de cálcio empregada como biomaterial para a reposição e regeneração óssea. O reconhecimento de suas limitações para o uso clínico, devido a sua lenta biodegradação, levou ao interesse crescente para a aplicação de outros fosfatos de cálcio, como o fosfato de cálcio amorfo (ACP), o fosfato octacálcico (OCP) e os tricálcio fosfatos (TCP). Assim, além da HA, os fosfatos de tricálcio (TCP) têm sido utilizados para fins de aplicação como biomateriais. O TCP pode ocorrer em quatro formas cristalinas: $\beta$-TCP; $\alpha$-TCP; $\alpha$-TCP e $\gamma$-TCP. 
Tabela I - Fosfatos de cálcio, fórmulas químicas e razões Ca/P $[1,3]$.

[Table I-Calcium phosfates, chemical formulas and Ca/P ratios [1, 3].]

\begin{tabular}{lcc}
\hline Fosfato de cálcio & Fórmula química & $\mathrm{Ca} / \mathrm{P}$ \\
Fosfato tetracálcico (TeCP) & $\mathrm{Ca}_{4} \mathrm{O}_{(}\left(\mathrm{PO}_{4}\right)_{2}$ & 2,0 \\
Hidroxiapatita (HA) & $\mathrm{Ca}_{10}\left(\mathrm{PO}_{4}\right)_{6}(\mathrm{OH})_{2}$ & 1,67 \\
Fosfato de cálcio amorfo (ACP) & $\mathrm{Ca}_{3}\left(\mathrm{PO}_{4}\right)_{2} \cdot n \mathrm{H}_{2} \mathrm{O}$ & 1,5 \\
Fosfato tricálcico $(\alpha, \alpha, \beta, \gamma)(\mathrm{TCP})$ & $\mathrm{Ca}_{3}\left(\mathrm{PO}_{4}\right)_{2}$ & 1,5 \\
Fosfato octacálcico (OCP) & $\mathrm{Ca}_{8} \mathrm{H}_{2}\left(\mathrm{PO}_{4}\right)_{6} \cdot 5 \mathrm{H}_{2} \mathrm{O}$ & 1,33 \\
Mono-hidrogênio fosfato de cálcio di-hidratado (DCPD) & $\mathrm{CaHPO}_{4} \cdot 2 \mathrm{H}_{2} \mathrm{O}$ & 1,0 \\
Mono-hidrogênio fosfato de cálcio (DCP) & $\mathrm{CaHPO}_{4}$ & 1,0 \\
Pirofosfato de cálcio (CPP) & $\mathrm{Ca}_{2} \mathrm{P}_{2} \mathrm{O}_{7}$ & 1,0 \\
Pirofosfato de cálcio di-hidratado (CPPD) & $\mathrm{Ca}_{2} \mathrm{P}_{2} \mathrm{O}_{7} \cdot 2 \mathrm{H}_{2} \mathrm{O}$ & 1,0 \\
Fosfato heptacálcico (HCP) & $\mathrm{Ca}_{7}\left(\mathrm{P}_{5} \mathrm{O}_{16}\right)_{2}$ & 0,7 \\
Di-hidrogênio fosfato tetracálcico (TDHP) & $\mathrm{Ca}_{4} \mathrm{H}_{2} \mathrm{P}_{6} \mathrm{O}_{20}$ & 0,67 \\
Fosfato monocálcico mono-hidratado (MCPM) & $\mathrm{Ca}_{2}\left(\mathrm{H}_{2} \mathrm{PO}_{4}\right)_{2} \cdot \mathrm{H}_{2} \mathrm{O}$ & 0,5 \\
Metafosfato de cálcio $(\alpha, \beta, \gamma)(\mathrm{CMP})$ & $\mathrm{Ca}_{2}\left(\mathrm{PO}_{3}\right)_{2}$ & 0,5 \\
\hline
\end{tabular}

A conversão de $\beta$-TCP em $\alpha$-TCP ocorre a pressão ambiente entre 1393 e $1453 \mathrm{~K}$; a conversão de $\alpha$-TCP em $\alpha$-TCP ocorre entre 1703 e $1743 \mathrm{~K}$. O aparecimento da fase $\gamma$-TCP só é possível sob condições de elevada pressão [1].

A Tabela I mostra os diversos tipos de fosfatos de cálcio e sua relação $\mathrm{Ca} / \mathrm{P}$. A relação $\mathrm{Ca} / \mathrm{P}$ é importante na avaliação da solubilidade destes compostos. De forma geral, quanto maior a relação $\mathrm{Ca} / \mathrm{P}$, menor será a solubilidade $[1,3]$. Fosfatos de cálcio com diferentes morfologias e estequiometrias podem ser preparados variando os parâmetros de síntese como: temperatura de precipitação, $\mathrm{pH}$ do meio, tempo reacional, velocidade de adição de reagentes, temperatura do tratamento térmico [6].

Resultados de testes clínicos sugerem que o $\beta$-TCP associado com implantes autólogos é um material promissor para o tratamento de lesões no maxilar [8]. O mesmo composto atua como material de auxilio para a regeneração óssea após a realização de implante odontológico $[9,10]$. Estudos mostram a utilização do $\beta$-TCP e de HA para capeamento pulpar, em substituição ao hidróxido de cálcio [11]. Os métodos mais simples para a síntese dos fosfatos de cálcio são baseados em reações químicas que agregam o íon cálcio ao íon fosfato, normalmente por via úmida e condições controladas de $\mathrm{pH}$. Os precursores mais utilizados são o $\mathrm{Ca}\left(\mathrm{NO}_{3}\right)_{2}, \mathrm{Ca}(\mathrm{OH})_{2}, \mathrm{H}_{3} \mathrm{PO}_{4},\left(\mathrm{NH}_{4}\right)_{2} \mathrm{HPO}_{4}$ e $\mathrm{NH}_{4} \mathrm{OH}$. Tratamentos térmicos são realizados para a obtenção de diferentes fases, como HA e as formas alotrópicas de TCP [12-14].

A utilização da casca de ovo é sugerida como uma alternativa para valoração de resíduos sólidos. A casca representa $10 \%$ do peso do ovo, gerando uma quantidade de resíduo de 5,92 milhões de toneladas por ano no mundo inteiro [15]. A produção de HA é sugerida como uma das alternativas viáveis, a partir da conversão do $\mathrm{CaCO}_{3}$ em $\mathrm{Ca}(\mathrm{OH})_{2}$, seguido por um ataque do ácido fosfórico sobre a base [15]. Esta técnica utiliza equipamentos e vidrarias simples, além de reagentes facilmente obtidos em laboratórios.

O presente trabalho descreve rotas químicas para a obtenção de $\beta$-TCP e outros fosfatos de cálcio, utilizando como precursor inicial a casca de ovo de galinha.

\section{MATERIAIS E MÉTODOS}

\section{Preparo da casca de ovo}

Separou-se o equivalente a $200 \mathrm{~g}$ de cascas de ovos de galinha obtidas do consumo doméstico. Todas se apresentavam totalmente limpas, com cor branca e livre de corpos estranhos em sua superfície. Após a coleta da matériaprima, as cascas dos ovos foram levadas ao triturador (Arno LE série HE), com $100 \mathrm{~mL}$ de água corrente para iniciar o processamento por $30 \mathrm{~s}$. Após a etapa de trituração, as cascas dos ovos foram peneiradas e levadas à fervura, com água destilada, durante $15 \mathrm{~min}$. Após a fervura, a matéria prima foi lavada com álcool $70 \%$ e levada para estufa para secagem por $24 \mathrm{~h}$ a $50{ }^{\circ} \mathrm{C}$. Finalizada a secagem, a matéria prima foi armazenada em recipiente de plástico esterilizado.

\section{Análise termodinâmica de reações ácidas sobre a casca de ovo}

O estudo termodinâmico, embasado na análise da variação da energia livre de Gibbs padrão $\left(\Delta G^{0}\right)$, foi conduzido com o auxílio do programa HSC 5.0. Este programa permite calcular os parâmetros termodinâmicos de uma reação química, como energia livre de Gibbs, variação de entalpia e entropia e a constante de equilíbrio. Foram estudadas as reações da casca do ovo com ácidos para a obtenção de uma rota química para a solubilização deste material. Neste estudo, foram avaliados os ácidos clorídrico $(\mathrm{HCl})$, nítrico $\left(\mathrm{HNO}_{3}\right)$, sulfúrico $\left(\mathrm{H}_{2} \mathrm{SO}_{4}\right)$, acético $\left(\mathrm{CH}_{3} \mathrm{COOH}\right)$ e fosfórico $\left(\mathrm{H}_{3} \mathrm{PO}_{4}\right)$ quando reagidos com o carbonato de cálcio presentes na casca do ovo. As reações 
descritas foram avaliadas pelo programa para a faixa de temperatura do sistema entre $273 \mathrm{~K}$ e $373 \mathrm{~K}$.

As reações abaixo representam os sistemas estudados termodinamicamente para o ataque do carbonato do cálcio, presente na casca de ovo, por diferentes ácidos:

$$
\begin{aligned}
& \text { I. } \mathrm{CaCO}_{3}+2 \mathrm{HCl}(\mathrm{aq}) \rightarrow \mathrm{CaCl}_{2}(\mathrm{aq})+\mathrm{CO}_{2}(\mathrm{~g})+\mathrm{H}_{2} \mathrm{O}(\mathrm{l}) \\
& \text { II. } \mathrm{CaCO}_{3}+2 \mathrm{HNO}_{3}(\mathrm{aq}) \rightarrow \mathrm{Ca}\left(\mathrm{NO}_{3}\right)_{2}(\mathrm{aq})+\mathrm{CO}_{2}(\mathrm{~g})+\mathrm{H}_{2} \mathrm{O}(\mathrm{l}) \\
& \text { III. } \mathrm{CaCO}_{3}+\mathrm{H}_{2} \mathrm{SO}_{4}(\mathrm{aq}) \rightarrow \mathrm{CaSO}_{4}+\mathrm{CO}_{2}(\mathrm{~g})+\mathrm{H}_{2} \mathrm{O}(\mathrm{l}) \\
& \text { IV. } \mathrm{CaCO}_{3}+2 \mathrm{CH}_{3} \mathrm{COOH}(\mathrm{aq}) \rightarrow \mathrm{Ca}\left(\mathrm{CH}_{3} \mathrm{COO}\right)_{2}(\mathrm{aq})+ \\
& \mathrm{CO}_{2}(\mathrm{~g})+\mathrm{H}_{2} \mathrm{O}(\mathrm{l}) \\
& \text { V. } 3 \mathrm{CaCO}_{3}+2 \mathrm{H}_{3} \mathrm{PO}_{4} \rightarrow \mathrm{Ca}_{3}\left(\mathrm{PO}_{4}\right)_{2}+3 \mathrm{CO}_{2}(\mathrm{~g})+3 \mathrm{H}_{2} \mathrm{O}(\mathrm{l})
\end{aligned}
$$

De acordo com a análise termodinâmica, os valores de $\Delta \mathrm{G}^{0}$ para a reação $\mathrm{V}$ oscilam entre -153 e $-205 \mathrm{~kJ}^{\mathrm{m}} \mathrm{mol}^{-1}$ entre as temperaturas de 273 e $373 \mathrm{~K}$. Estes valores de $\Delta \mathrm{G}^{0}$ indicam, do ponto de vista termodinâmico, que a reação $\mathrm{V}$ é a mais favorável para a obtenção de íons cálcio no meio reacional. Embora menos favoráveis, todas as demais reações também apresentam boa espontaneidade para a conversão do carbonato de cálcio em íons cálcio, visto que as mesmas apresentam valores de $\Delta \mathrm{G}^{0}$ negativos na faixa de temperatura considerada. A reação I apresenta valores de $\Delta \mathrm{G}^{0}$ entre $-56 \mathrm{~kJ}$ e $-74 \mathrm{~kJ}$. mol ${ }^{-1}$; a reação II apresenta valores entre $-37 \mathrm{~kJ}$ e $-40 \mathrm{~kJ}^{\mathrm{mol}}{ }^{-1}$; a reação III mostrou valores entre $-135 \mathrm{~kJ}$ e $-149 \mathrm{~kJ}^{\mathrm{mol}}{ }^{-1}$ e a reação IV entre $-13 \mathrm{~kJ} \mathrm{e}-14 \mathrm{~kJ}$. $\mathrm{mol}^{-1}$ tem os valores mais positivos de $\Delta \mathrm{G}^{0}$.

\section{Rotas para a síntese de fosfatos de cálcio}

Todas as reações descritas foram testadas em laboratório. Em 5 béqueres de $1000 \mathrm{~mL}$, sob agitação constante, foram adicionados $500 \mathrm{~mL}$ dos ácidos na concentração de $1 \mathrm{M}$ a 26,8 g de casca de ovo triturada. A digestão química foi bem sucedida para todos os ácidos, sendo finalizadas em 2 h, com exceção do ácido acético, sendo completada após 24 h. Os ácidos clorídrico e nítrico dissolveram totalmente a casca do ovo, não havendo resíduos após a finalização do processo. As reações com os ácidos sulfúrico e fosfórico levaram a formação de precipitados de sulfato de cálcio e fosfato de cálcio, respectivamente. $\mathrm{O}$ ácido acético também gerou uma solução sem a presença de precipitados, após um longo tempo de reação $(24 \mathrm{~h})$. A partir dos produtos reacionais presentes nos bécheres com $\mathrm{HCl}, \mathrm{HNO}_{3}$ e $\mathrm{H}_{3} \mathrm{PO}_{3}$ foram estabelecidas as rotas para a obtenção de derivados de fosfato de cálcio descritas a seguir: (1) rota via utilização do $\mathrm{HCl}$ com conversão para $\mathrm{Ca}(\mathrm{OH})_{2}$ e reação com $\mathrm{H}_{3} \mathrm{PO}_{4}$; (2) rota via utilização de $\mathrm{HCl}$ com reação com $\mathrm{Na}_{2} \mathrm{HPO}_{4}$; (3) rota via utilização do $\mathrm{HNO}_{3}$ e reação com $\mathrm{H}_{3} \mathrm{PO}_{4}$; (4) rota via utilização do $\mathrm{H}_{3} \mathrm{PO}_{4}$ sobre a casca de ovo.

(1) Rota via utilização do $\mathrm{HCl}$ com conversão para $\mathrm{Ca}(\mathrm{OH})_{2}$ e reação com $\mathrm{H}_{3} \mathrm{PO}_{4}$

Em um béquer contendo $500 \mathrm{~mL}$ da solução de $\mathrm{CaCl}_{2} \cong$ $28 \mathrm{~g}$ do sal dissolvido) obtida na reação I, foram adicionados lentamente $20 \mathrm{~g}$ de $\mathrm{NaOH} 0,5 \mathrm{M}$ sob agitação e temperatura constantes $\left(50{ }^{\circ} \mathrm{C}\right)$ durante $1 \mathrm{~h}$. O sistema foi mantido em $\mathrm{pH}=10$. Ao final, verificou-se a formação de um precipitado branco, sendo posteriormente lavado com água deionizada, filtrado a vácuo e levado à estufa para secagem a $100{ }^{\circ} \mathrm{C}$ por $24 \mathrm{~h}$. Ao final desse procedimento obteve-se a massa de 6,24 g de um fino pó branco, identificado por DRX como $\mathrm{Ca}(\mathrm{OH})_{2}$. $\mathrm{O} \mathrm{Ca}(\mathrm{OH})_{2}$ foi adicionado a $150 \mathrm{~mL}$ de ácido fosfórico $1 \mathrm{M}$ no interior de um béquer de $500 \mathrm{~mL}$. O sistema foi mantido sob agitação constante, a $50{ }^{\circ} \mathrm{C}$ e com $\mathrm{pH}=10$, controlado via adição de $\mathrm{NH}_{4} \mathrm{OH} 1 \mathrm{M}$. Ao final do procedimento, foi obtido um sólido branco, finamente dividido no meio reacional. O sólido obtido foi lavado com água deionizada, filtrado e levado para secagem em estufa a $100{ }^{\circ} \mathrm{C}$ por $24 \mathrm{~h}$, obtendo uma massa final de $9,73 \mathrm{~g}$. Este pó foi levado a tratamento térmico a $900{ }^{\circ} \mathrm{C}$ durante $2 \mathrm{~h}$. A Fig. 1 resume os procedimentos reacionais da rota via $\mathrm{HCl}$.

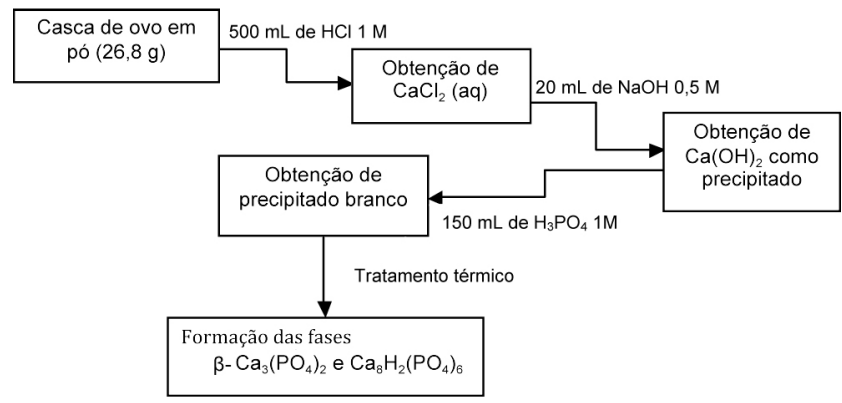

Figura 1: Rota de síntese utilizando $\mathrm{HCl}, \mathrm{NaOH}$ e $\mathrm{H}_{3} \mathrm{PO}_{4}$ como reagentes.

[Figure 1: Synthesis route using $\mathrm{HCl}, \mathrm{NaOH}$ and $\mathrm{H}_{3} \mathrm{PO}_{4}$ as reactants.]

(2) Rota via utilização do $\mathrm{HCl}$ com reação com $\mathrm{Na}_{2} \mathrm{HPO}_{4}$

Em um béquer contendo $500 \mathrm{~mL}$ da solução de $\mathrm{CaCl}_{2} \cong$ $28 \mathrm{~g}$ do sal dissolvido) obtida na reação I, foram adicionados lentamente $21,30 \mathrm{~g}$ de $\mathrm{Na}_{2} \mathrm{HPO}_{4}$ sob agitação e temperatura constante $\left(50{ }^{\circ} \mathrm{C}\right)$. A reação foi processada por $1 \mathrm{~h} \mathrm{sem}$ controle de $\mathrm{pH}$. Após o período estipulado para a reação, verificou-se a formação de um precipitado branco, sendo posteriormente lavado com água deionizada, filtrado a vácuo e levado à estufa para secagem a $100{ }^{\circ} \mathrm{C}$ por $24 \mathrm{~h}$. Obtevese a massa de $17,91 \mathrm{~g}$ de pó branco que foi submetido a tratamento térmico a $900{ }^{\circ} \mathrm{C}$ durante $2 \mathrm{~h}$. A Fig. 2 mostra os procedimentos realizados nessa rota de síntese.

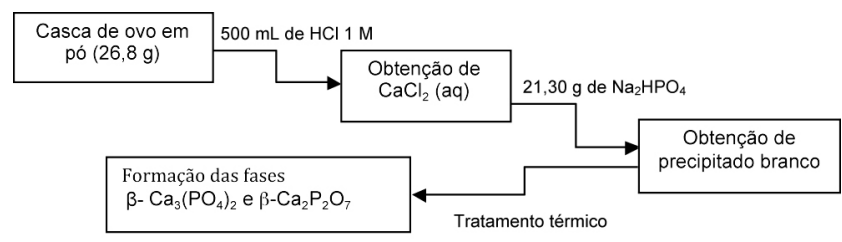

Figura 2: Rota de síntese através de reação com $\mathrm{HCl}$ e $\mathrm{Na}_{2} \mathrm{HPO}_{4}$. [Figure 2: Synthesis route using $\mathrm{HCl}$ and $\mathrm{Na}_{2} \mathrm{HPO}_{4}$ as reactants.] 


\section{(3) Rota via utilização do $\mathrm{HNO}_{3}$}

Em um béquer contendo $250 \mathrm{~mL}$ da solução de $\mathrm{Ca}\left(\mathrm{NO}_{3}\right)_{2}$

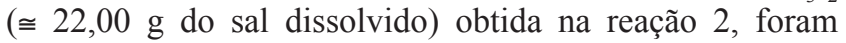
adicionados lentamente $19,03 \mathrm{~g}$ de $\mathrm{Na}_{2} \mathrm{HPO}_{4}$ sob agitação e temperatura constante $\left(50^{\circ} \mathrm{C}\right)$. A reação foi processada por um período de $1 \mathrm{~h}$ sem controle de $\mathrm{pH}$. Ao término da reação, obteve-se uma massa de 7,9 g de um precipitado branco, sendo posteriormente lavado com água deionizada, filtrado a vácuo e levado à estufa para secagem a $100^{\circ} \mathrm{C}$ por $24 \mathrm{~h}$. Este material foi levado a tratamento térmico a $900^{\circ} \mathrm{C}$ durante 2 h. A Fig. 3 mostra os procedimentos da rota via $\mathrm{HNO}_{3}$.

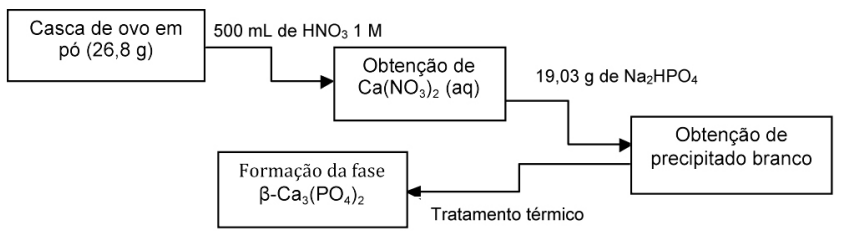

Figura 3: Rota de síntese utilizando $\mathrm{HNO}_{3}$ e $\mathrm{Na}_{2} \mathrm{HPO}_{4}$ como reagentes.

[Figure 3: Synthesis route using $\mathrm{HNO}_{3}$ and $\mathrm{Na}_{2} \mathrm{HPO}_{4}$ as reactants.]

\section{Medidas de difração de raios $X(D R X)$}

As análises por difração de raios $\mathrm{X}$ foram realizadas nas amostras obtidas na forma de pó, em difratômetro Siemens D5000 com radiação Cuk $\alpha$ (1,5453 $)$ com uma tensão de tubo $40 \mathrm{kV}$, corrente $45 \mathrm{~mA}$ e varredura entre $5,0^{\circ} \mathrm{e} 90,0^{\circ}$. O tempo de coleta foi $0,05^{\circ} . \mathrm{s}^{-1}$ por passo. Para a identificação das fases foi utilizado o programa EVA com banco de dados JCPDF.

\section{RESULTADOS}

A análise por DRX do material obtido, via utilização de $\mathrm{HCl}$ e conversão posterior em $\mathrm{Ca}(\mathrm{OH})_{2}$, de acordo com a rota descrita em (1), após tratamento térmico indicou a presença das fases $\beta-\mathrm{Ca}_{3}\left(\mathrm{PO}_{4}\right)_{2}$ e de $\mathrm{Ca}_{8} \mathrm{H}_{2}\left(\mathrm{PO}_{4}\right)_{6}$. A Fig. 4 mostra o DRX deste material, onde os picos de difração da fase $\beta-\mathrm{Ca}_{3}\left(\mathrm{PO}_{4}\right)_{2}$ estão indicados por (1) e da fase $\mathrm{Ca}_{8} \mathrm{H}_{2}\left(\mathrm{PO}_{4}\right)_{6}$ estão indicados por (2).

A segunda via, através do ataque do $\mathrm{HCl}$ à casca de ovo e reação do produto formado com $\mathrm{Na}_{2} \mathrm{HPO}_{4}$ levou à formação de duas fases distintas correspondendo à formação do $\beta$-tricálcio fosfato $\left[\left(\mathrm{Ca}_{3}\left(\mathrm{PO}_{4}\right)_{2}\right]\right.$ e do $\beta$-pirofosfato de cálcio $\left(\beta-\mathrm{Ca}_{2} \mathrm{P}_{2} \mathrm{O}_{7}\right)$, conforme mostrado no DRX da Fig. 5 onde os picos de difração da fase $\beta-\mathrm{Ca}_{3}\left(\mathrm{PO}_{4}\right)_{2}$ estão indicados por (1) e da fase $\beta$-pirofosfato de cálcio $\left(\beta-\mathrm{Ca}_{2} \mathrm{P}_{2} \mathrm{O}_{7}\right)$ estão indicados por (2).

A outra via estudada, através do ataque de $\mathrm{HNO}_{3}$ sobre a casca do ovo, também foi analisada por DRX. O resultado do pó, após tratamento térmico, mostrou a presença somente da fase $\beta-\mathrm{Ca}_{3}\left(\mathrm{PO}_{4}\right)_{2}$. A Fig. 6 mostra o DRX deste material, mostrando a fase única resultante do processamento descrito.

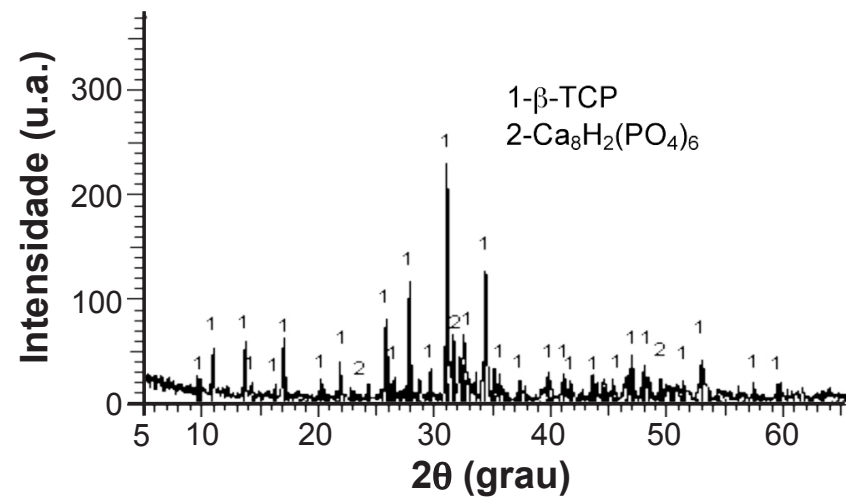

Figura 4: DRX do material obtido pela rota que utiliza $\mathrm{HCl}, \mathrm{NaOH}$ e $\mathrm{H}_{3} \mathrm{PO}_{4}$ como reagentes. Os picos de difração da fase $\beta-\mathrm{Ca}_{3}\left(\mathrm{PO}_{4}\right)_{2}$ estão indicados por (1) e da fase $\mathrm{Ca}_{8} \mathrm{H}_{2}\left(\mathrm{PO}_{4}\right)_{6}$ estão indicados por (2). [Figure 4: XRD pattern of material obtained from $\mathrm{HCl}, \mathrm{NaOH}$ and $\mathrm{H}_{3} \mathrm{PO}_{4}$ synthesis route. The diffraction plane peaks are indicated as: $\beta-\mathrm{Ca}_{3}\left(\mathrm{PO}_{4}\right)_{2}$ (1) and $\mathrm{Ca}_{8} \mathrm{H}_{2}\left(\mathrm{PO}_{4}\right)_{6}(2)$ phases.]

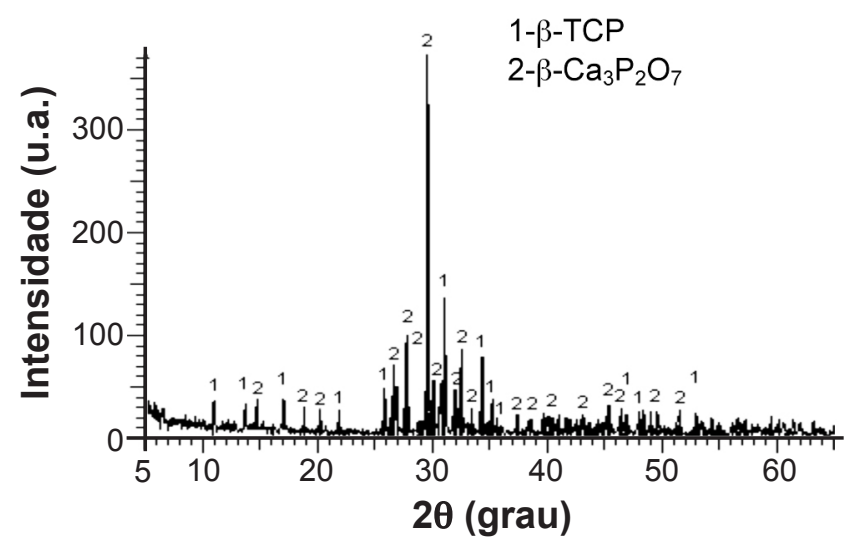

Figura 5: DRX do material obtido a partir da rota que utiliza $\mathrm{HCl}$ e $\mathrm{Na}_{2} \mathrm{HPO}_{4}$. Os picos de difração da fase $\beta-\mathrm{Ca}_{3}\left(\mathrm{PO}_{4}\right)_{2}$ estão indicados por (1) e da fase $\beta$-pirofosfato de cálcio $\left(\beta-\mathrm{Ca}_{2} \mathrm{P}_{2} \mathrm{O}_{7}\right)$ estão indicados por (2).

[Figure 5: XRD pattern of material obtained from $\mathrm{HCl}$ and $\mathrm{Na}_{2} \mathrm{HPO}_{4}$ synthesis route. The diffraction plane peaks are indicated as: $\beta-\mathrm{Ca}_{3}\left(\mathrm{PO}_{4}\right)_{2}(1)$ and $\left(\beta-\mathrm{Ca}_{2} \mathrm{P}_{2} \mathrm{O}_{7}\right)(2)$ phases.]

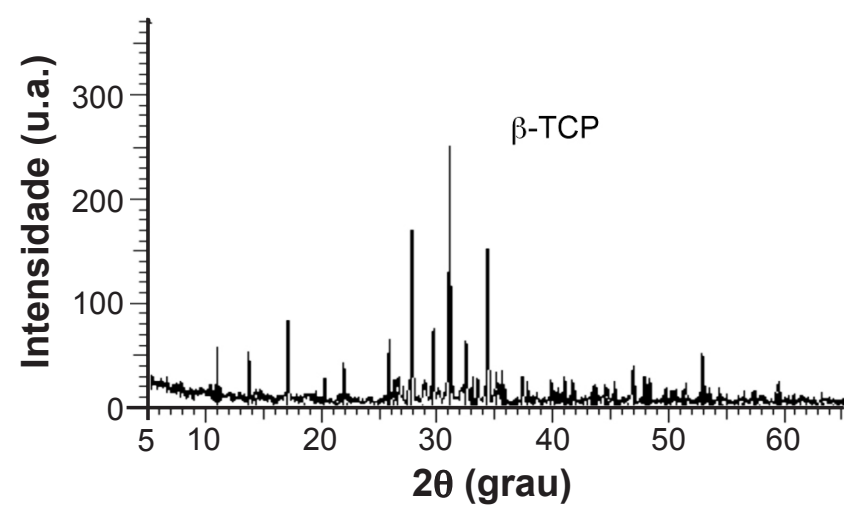

Figura 6: DRX do material obtido a partir da rota que $\mathrm{HNO}_{3}$ e $\mathrm{Na}_{2} \mathrm{HPO}_{4}$ como reagentes. Todos os picos de difração presentes pertencem a fase $\beta$-TCP.

[Figure 6: XRD pattern of material obtained from $\mathrm{HCl}$ and $\mathrm{Na}_{2} \mathrm{HPO}_{4}$ synthesis route. The all XRD diffraction peaks indicated the presence of pure $\beta$-TCP phase.] 


\section{CONCLUSÕES}

A investigação de rotas químicas para a obtenção de fosfatos de cálcio a partir da casca de ovo de galinha mostrou a viabilidade dos processos para as via reacionais pesquisadas. A obtenção dos diferentes tipos de fosfatos no meio pode ser atribuída aos parâmetros reacionais como: tipo de reagentes, temperatura, $\mathrm{pH}$ do meio, tempo de reação e precipitação. A primeira e a segunda rotas investigadas resultaram a formação de diferentes fases de fosfatos de cálcio. Enquanto a primeira rota mostrou a presença de $\beta$-TCP juntamente com $\mathrm{Ca}_{8} \mathrm{H}_{2}\left(\mathrm{PO}_{4}\right)_{6}$, a segunda rota apresentou além da fase $\beta$-TCP, a formação de $\beta-\mathrm{Ca}_{2} \mathrm{P}_{2} \mathrm{O}_{7}$. A terceira rota estudada, utiliza o sal $\mathrm{Ca}\left(\mathrm{NO}_{3}\right)_{2}$, obtido da reação do ácido nítrico com a casca de ovo. Essa rota foi capaz de produzir apenas a fase $\beta$-TCP no meio reacional. Do ponto de vista de processo para a síntese de biomateriais, a terceira rota foi a mais adequada, pois permite a produção de uma fase única de fosfato de cálcio no meio ( $\beta$-TCP). Conforme citado, esse material apresenta crescente relevância por sua aplicação como substituto ósseo.

\section{REFERÊNCIAS}

[1] A. C. Guastaldi, A. H. Aparecida, Química Nova 33, 6 (2010) 1352-1358.

[2] M. H. Santos, L. G. D. Eneide, H. S. Mansur, Anais $17^{\circ}$ CBECIMAT, Foz do Iguaçu, PR (2006).

[3] A. C. F. M. Costa, M. G. Lima, V. V. Cordeiro, K. M. S.
Viana, C. V. Souza, H. L. Lira, Rev. Eletr. Mater. Proc. 43 (2009) 29-38.

[4] B. C. S. Rigo, S. A. Gehrke, M. Carbonari, Rev. Dental Press Periondontia Implantol. 1, 3 (2007) 39-50.

[5] J. C. Araújo, L. Sena, I. N. Bastos, G. D. A. Soares, Química Nova 30, 8 (2007) 1853-1859.

[6] L. M. Rodriguez-Lorenzo, M. Vallet-Regí, Chem. Mater. 12 (2000) 2460-2465.

[7] E. Y. Kawachi, C. A. Bertran, R. R. Reis, O. L. Alves, Química Nova 23 (2000) 518-522.

[8] L. A. A Zorzano, M. J. R. Tojo, J. M. A. Urizar, Med. Oral Patol. Oral Cir. Bucal. Nov 1 12,7 (2007) E-532-536.

[9] J. P. Mourelo, A. J. Guerra, L. M. Guil, J. J. S. Egea, E. V. Ortega, Av. Periodoncia 22, 3 (2010) 127-137.

[10] E. V. Ortega, J. P. Mourelo, J. J. S. Egea, O. P. Perez, R. M. Soteras, Av. Periodoncia 19, 3 (2007) 141-149.

[11] C. S. Delfino, C. Ribeiro, G. F. Vieira1, A. H. A. Bressiani, M. L. Turbino, Cerâmica 56, 340 (2010) 381-388. [12] A. C. B. M. Fook, A. H. Aparecida, M. V. L. Fook, Rev. Matéria 15, 3 (2010) 392-399.

[13] A. L. Rosa, M. Y. Shareef, R. V. Noort, Pesq. Odont. Brás. 14, 3 (2000) 273-277.

[14] R. G. Carrodegas, L. M. Alonso, J. A. D GarcíaMenocal, L. M. Alonso, M. P. G. Molins, S. M. Manent, J. G. Mur, J. T. Pérez, J. A. P. Estany, Mater. Res. 6, 3 (2003) 395-401.

[15] D. A. Oliveira, P. Benelli, E. R. Amante, $2^{\text {nd }}$ Int. Workshop Adv. Cleaner Prod., S. Paulo, SP, Brasil, 1-11 (2009).

(Rec. 12/06/2011, Rev. 17/02/2012, Ac. 17/04/2012) 